\title{
Charging Electric Vehicles Using Opportunistic Stopovers
}

\author{
Pierluigi Gallo \\ DEIM / CNIT \\ Università di Palermo, viale delle Scienze ed. 9 \\ Palermo, 90128, Italia \\ pierluigi.gallo@unipa.it
}

\begin{abstract}
The diffusion of electric vehicles asks for efficient energy replenishment, which requires geographical and temporal coordination of shared charging resources. We introduce a novel charging methodology that exploits users' opportunistic mobility. This paper focuses on vehicle stopovers detecting potential charging opportunities. Our mobility-assisted methodology protects users privacy and permits a hybrid centralized/distributed approach avoiding clashes with other potential users. A preliminary analysis on our charging system, obtained with mobility data from the field, shows that among the available charging stations, some are more relevant and have a key role in serving electric vehicle recharge. This can be useful for further investigation on designing charging networks and aggregating electric vehicles towards charging stations.
\end{abstract}

\section{INTRODUCTION}

In recent years several models of Electric Vehicles (EVs) with low environmental impact appeared in the market and new charging stations have been deployed in major cities allowing electric mobility to be a valid alternative to a traditional one.

Electric mobility introduces nomadic consumption in contrast to fixed energy drainage associated to physical places. Instead of using one single point of delivery (POD), electric mobility requires several PODs for one single user, therefore a shared infrastructure is required. In fact, electric vehicles are mobile loads with consumption profiles that differ from residential loads (in time, power, and position). Unlike domestic PODs, which are dedicated to the households, charging stations are shared resources that serve independent drivers for recharging their electric vehicles.

Compared to refueling, the energy replenishment for electric vehicles may require a significant amount of time depending on the battery (capacity and state of charge) and on the charging station (technology and power). Additionally, stations for electric vehicles are not as pervasively distributed in space as petrol ones, therefore charges may require dedicated transfers. These time and spatial constraints have to be adequately taken into account while designing recharging systems for EVs because they can have a significant impact on drivers' mobility needs.

In principle, users can recharge their EVs all the time these vehicles are off at stopovers (e.g. while the user is at work, shopping, even while stopping at the lights in case of wireless charging systems). However, charges are significant only when the duration of stopovers is long enough and are convenient when the user's destination location is at walking distance from a free charging station. These facts motivated us to focus on users' mobility, exploiting it for computing optimal charging stations considering users' mobility profile in space and time.

The analysis of mobility is beneficial for electric charging: actual movements help to predict energy consumption and have been extensively studied in literature (e.g. [1], [2]), generally joined to the monitoring of battery state of charge (SoC). However, the analysis of stopovers appears to be underexplored, despite it can be helpful for finding the possibilities to recharge EVs, named in the following as charging opportunities. These arise when time and stopover position are convenient for charging. In fact, previous studies on mobility generally take into account actual changes of position over time. We also analyze the starting time, duration and position of stopovers. Furthermore, in our analysis we consider two mobility components: (i) movements to satisfy users' needs, i.e. the intended mobility, which we opportunistically exploit; (ii) movements to fulfill context requirements, as driving in search of parking and charging stations that we call metamobility.

Drivers move with their vehicles to satisfy their mobility needs (we want to exploit such opportunistic mobility without interfering with it) and to satisfy vehicles needs (roaming while looking for parking, charging stations, etc.). Our charging system aims to minimize the impact on the intended mobility, to use it opportunistically and minimize meta-mobility. This requires, as already indicated before, an analysis in time and space of vehicle movements and stopovers.

Our system provides charging recommendations that maximize the utility for users (who save time and money) and for the Distribution System Operator (DSO) (who reduces peak loads caused by uncontrolled EV loads [3]). This mobilitybased approach is complementary to DSOs strategies that tend to govern peak loads and reduce technical losses by the mean of dynamic billing rates during the time of day, rising prices for discouraging consumption [4].

The orchestration of such charging system is demanded to 
a central coordination that updates the status of the system in time and space. Our centralized control coordinates the use of emerging charging opportunities and avoids clashes in the access to shared resources.

However, providing mobility information to a central entity inevitably introduces privacy issues, mostly related to the disclosure of user's position. We reduce the information centrally given to the coordination system by exposing only aggregated statistics. Sensitive details on user's mobility such as positions, trajectories and timings are kept locally on her smartphone, which is assumed to be the heart of our sensing system.

\section{RELATED WORK}

Charging EVs has been studied in literature from the DSO and the vehicle perspectives. Charges have been optimized to maximize the benefits for the DSO, preventing the distribution network from overloading and reducing the total costs due to inefficiencies and losses [5]. On the other side charges have been optimized keeping in mind benefits for the vehicle and its battery [6].

EVs are energy storage elements, which can either consume or inject power to the grid, under the Vehicle-to-Grid paradigm (V2G) [7], [8]. V2G permits leveling peak loads, by charging at night when demand is low and sending power back to the grid during peak hours (typically daytime). Distributors use economic incentives to motivate EV users and orient their consumption. The authors in [9] proposed two algorithms based on a forecast of future electricity prices and use dynamic programming to find the economically optimal solution for the vehicle owner. In [10] there are explored both the centralized and decentralized approaches. In the first case, a software inside the EV decides when to plug the EV for charging. The algorithm uses information obtained from EV owners, such as where and how long their vehicles will remain parked and their prevision about energy consumption, for generating a control price signal in case of congested network. In the centralized approach scenario, a central utility controller could determine for the EVs when to charge, relying on information similar to the previous case but accordingly to a centralized decision. One widely used method for coordinating EV charges is to consider the aggregation of their loads. This entity clusters and controls many vehicles and offers ancillary services like smart charging and V2G services [11], [12]. One of the issues recently investigated is the mobility of EV. In [13] it was proposed a mobility-aware coordinated charging strategy for EVs. Such approach uses EV position to communicate in realtime the charging decision to the driver, through a vehicular ad-hoc network (VANET) smart grid that supports real-time communications to and from vehicles.

The works indicated above do not take into account the habitual user mobility, which have a central role in our approach. Our charging policy preserves the characteristics of users' intended mobility and does not require specific diversions, unless the urgency of charge requires an immediate intervention.

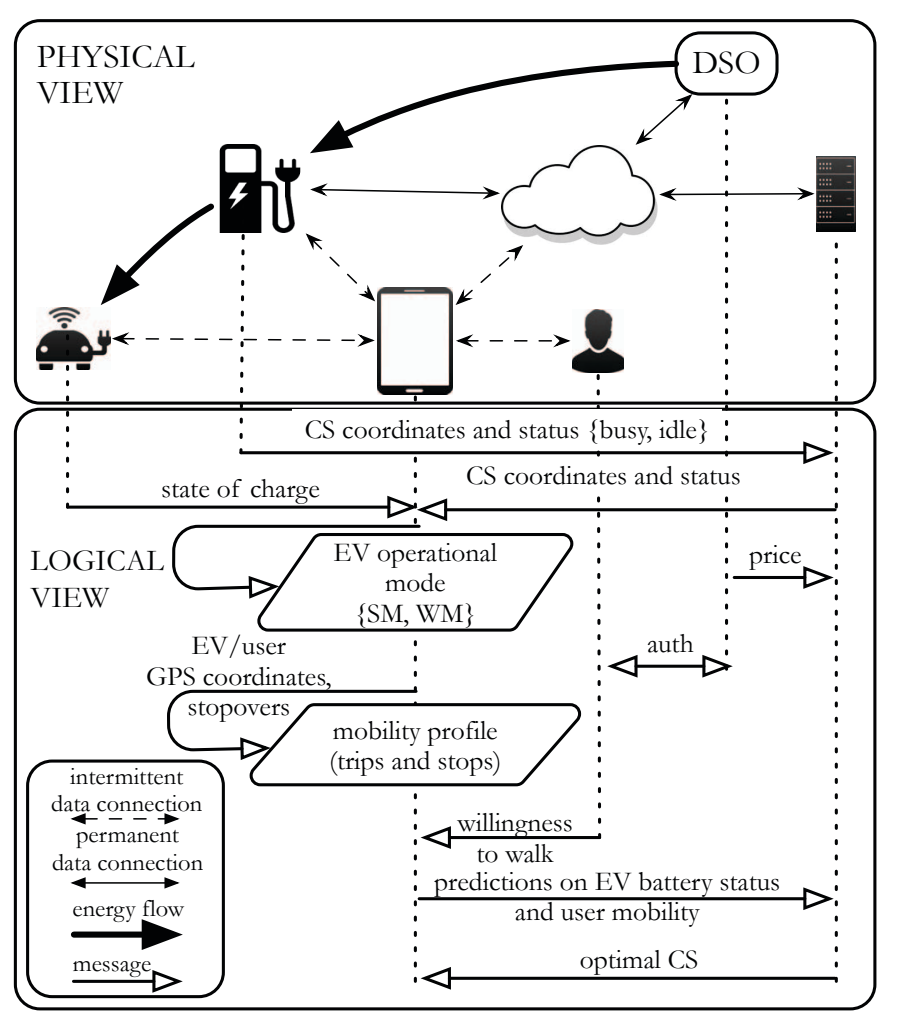

Fig. 1. System architecture with distributed location intelligence on smartphones and centralized optimization on the cloud.

This paper provides also a model for optimizing EV charges taking into account user mobility, positions of charging stations and their energy prices. Mobility analysis for charging EVs generally focuses on the definition and detection of regular paths and user habits. These were resumed by routine trips, evaluated by clustering routes accordingly to their similarities [14]. We do not infer staypoints from routing trips, we rather focus on stopovers directly from raw traces, fitting better the needs of our charging goals.

\section{SYSTEM DESCRIPTION}

The EV is assumed to have low-range communication capabilities in order to share data and messages with the driver's smartphone. The smartphone should be connected to the Internet at least temporarily, it provides the interfaces between our charging system and the final user and knows the evolution of EV operational mode, driver's position and habitual routes.

The proposed charging system collects data from different sources and mixes centralized optimization with distributed location intelligence to avoid disclosing user position and therefore preserving user privacy. Fig. 1 reports the system components: on the top it is presented the physical view with topological connections. On the bottom there is the logical view explaining the message flow.

- Charging stations are characterized by the coordinates of their positions and by their busy or idle status. Charg- 
ing stations are able to communicate with the driver's smartphone.

- Smartphones record user trajectories and analyze their mobility data in both dynamic and still components: trips and stopovers. Traveled distance, altimetry profiles, and consumed energy are key metrics for trips. Stopovers are characterized by location coordinates, starting time and duration. Smartphones and EVs are considered co-located until they are paired via Bluetooth technology, otherwise their positions may differ. The smartphone provides distributed location intelligence and protects user privacy by sharing only statistics and specific predictions with selected and trusted entities.

- Users provide their willingness to divert the desired path for parking close to a charging station, from now on indicated as willingness to walk. This value cannot be controlled by the system, it is explicitly provided by the user and depends on exogenous factors (mood, health, time constraints, etc.).

- EVs provide their SoC and internal energy status (charging, maintaining, discharging).

- The DSO energizes charging stations and provides price signals. These signals have heterogeneous values depending on the CS location. The user authenticates towards the DSO and, in case of success, the power flow is enabled.

- Our central system knows energy prices and CS positions as well as predictions regarding user mobility, battery status and user willingness to walk. Such information is used to periodically run an optimization algorithm and provide suggestions on the optimal charging station.

EVs shift between two operational modes. EVs work in safe mode (SM) when the charge is enough to guarantee the next trip(s) with a certain safety margin. In safe mode the optimal charging solutions is suggested, taking into account user opportunistic mobility. In warning mode (WM), the battery residual energy is below a threshold and charges cannot be deferred without the risk to be left on foot. The user is suggested to stop and immediately charge at a set of possible charging stations.

\section{USER MOBILITY}

Users roam in the urban environment depending only on their mobility needs. Most people show periodic mobility patterns, predictable together with their consequent charging opportunities.

Several data sets have been used for analyzing charging opportunities both in space and time. The D4Science repository contains vehicle mobility traces. The location of charging stations have been obtained from ChargeMap, a communitydriven map of charging points. We focus on two cities in Tuscany, Pisa and Pontedera, because of the joint availability of charging stations and mobility data in that area.

Mobility trajectories have been extracted from the raw sequences of positions, these have been measured every few dozens of seconds. Two exemplary trajectories of independent vehicles are reported in Fig. 2. The green trajectory shows

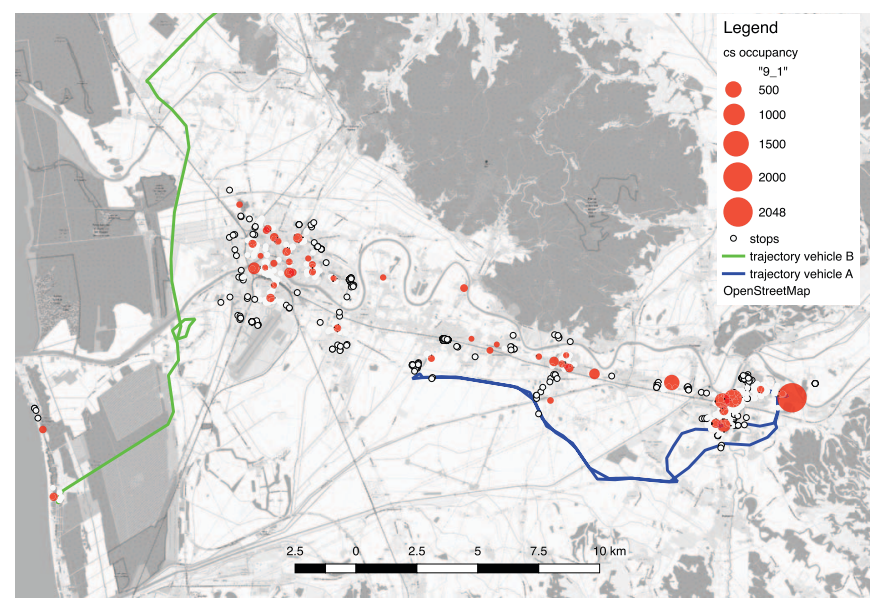

Fig. 2. Charging station occupancy exploiting our opportunistic mobility approach using real vehicle trajectories.

a path that comes from the North, skirts the Pisa urban area, then stops on the seaside at walking distance from a charging station. The blue trajectory depicts a loop close to Pontedera center. This small town contains several stopovers in a restricted area, due to there is an important industrial center and the city rail station.

Stops are shown in figure with white circles, charging stations with red dots, their size indicates the number of opportunistic stopovers that can be used as charging opportunities over that specific station. Stops that are at walking distance from one charging station are taken into account. The idea is that the user parks the EV at the charging station then walks till the intended destination. Therefore, the diversion of the intended trajectory is limited.

Stops can be considered charging opportunities only in case they are proximal to at least one charging station. The urban area has been partitioned by the mean of a Voronoi diagram over positions of charging stations. Then, CSs have been assigned with the number of potential charging opportunities, given by stops within the corresponding Voronoi region.

The opportunistic mobility of 81 vehicles has been analyzed. Despite such vehicles are not electric, we reasonably assume that the intended mobility does not change with the type of fuel. This assumption is not valid for meta-mobility, while roaming in search of a free charging station.

\section{THE CHARGING MODEL}

The evolution of the energy status of the EV is modeled by the mean of nested finite state machines, as reported in Fig. 3. Batteries of EVs are drained during trips (i.e. when the vehicle is $\mathrm{ON}$ ) and can maintain their charge or being recharged when the vehicle stops (i.e. when it is OFF). To better exploit charging opportunities, the vehicle is plugged to the CS after its stop and is unplugged at its departure. However, the station can postpone powering the vehicle in order to match DSO constraints. We do not continuously monitor the state of charge of the EV batteries, we only 


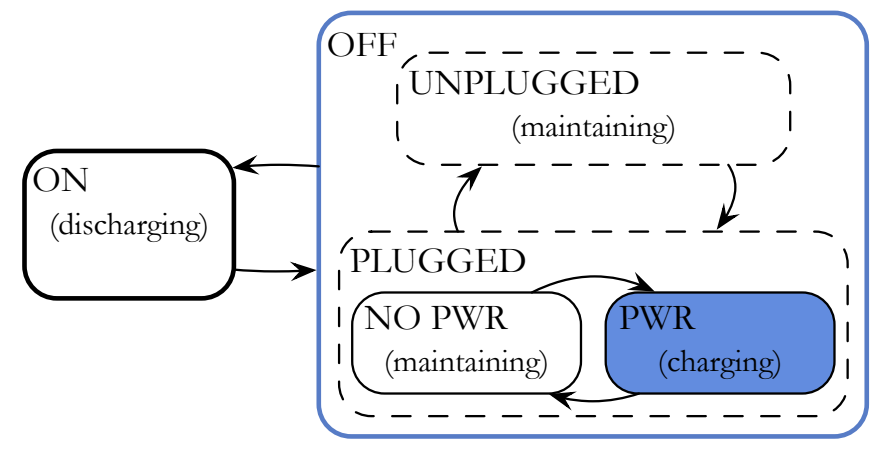

Fig. 3. EV status evolves as modeled by nested finite state machines. When the EV is off, potential charging opportunities arise (box with blue frame). If the vehicle is also plugged to the charging station outlet and powered (blue box), then this opportunity is realized and the EV actually charges.

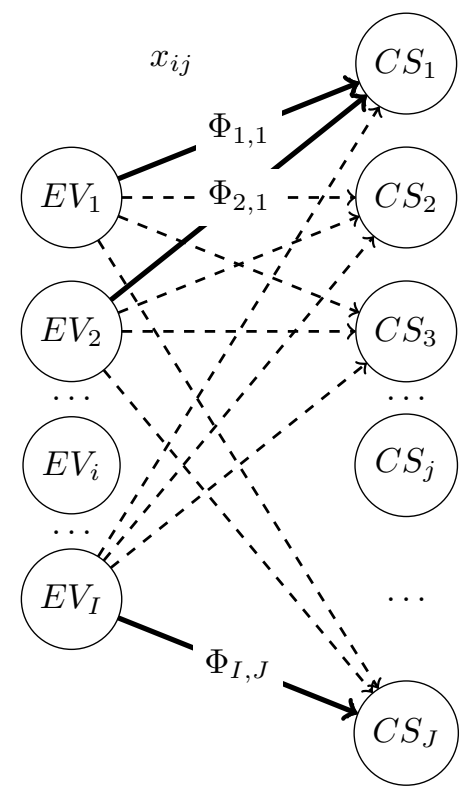

(a)

Fig. 4. Model for assigning EVs to charging stations depending on the extra distance $d_{i j}$ till the charging station and the price of energy.

consider an alarm in case the residual energy goes below a safety threshold; such functionality is supported by most of current EV models.

The assignment of EVs to charging stations is suggested by the centralized system using the model indicated in Fig. 4, which permits coordinating charging opportunities. Every time the user is going to stop close enough to a free charging station, the costs $\Phi_{i j}$ are computed, depending on the extra distance of the transfer till the CS and the energy price the CS currently offers.

In this bipartite graph, we indicate electric vehicles on the left $(i \in\{1, \ldots, I\})$ and $J$ charging stations on the right. When a new charging opportunity arises, the centralized algorithm receives the coordinates of the intended stop. The system knows the status of charging stations, if they are busy or idle,

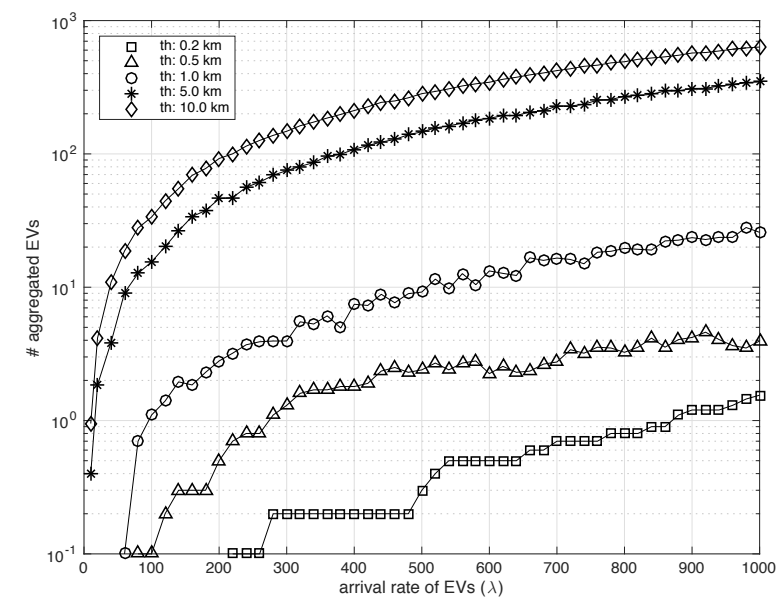

(a)

Fig. 5. Number of electric vehicles that can be aggregated to charging stations depending on their arrival rate, changing the maximum willingness to walk for users.

and the expected parking duration, obtained from the mobility profile and minimizes the following cost function:

$$
\min _{x_{i j}, a_{i j}} \sum_{i=1}^{I} \sum_{j=1}^{J} \Phi_{i j}
$$

Cost associated to arcs contain two components: $\Phi_{i j}=p_{j} x_{i j}+a_{i j} d_{i j}$. First, the cost of the electric energy, obtained considering the unit cost $p_{j}$ per $\mathrm{kWh}$ at the $\mathrm{j}$-th charging station times $x_{i j}$, the energy provided to the i-th EV (in $\mathrm{kWh}$ ) during its charge at the $\mathrm{j}$-th $\mathrm{CS}$.

The second component includes the cost due to the traveled distance $d_{i j}$ from the intended stop till the optimal charging station. This cost has to be taken into account only if $E V_{i}$ actually goes to recharge at $C S_{j}$. This is modeled through binary variables $a_{i j} \in\{0,1\}$ that is 1 only in case the path is effectively used. From the explanation above it results that $\sum_{i \in I} a_{i j} \leq 1$ because EVs can recharge only at one CS. This boundary is also shown in the bipartite graph: EVs can have at most one going out bold arrow. CSs are equipped with multiple sockets, whose quantity is $s_{j}$ for the $\mathrm{j}$-th charging station, which can host and charge more vehicles at one time, having $\sum_{j \in J} a_{i j} \leq s_{j}$.

Since CS have multiple sockets, we further model charging stations as multi-servant queuing systems where servants are the outlets and clients are the EVs. Under such assumptions, indicating the average duration of parking times with $\bar{P}$, the average charging arrival rate $\lambda$ and the average number of charging EVs $N_{E V}$, the Little's theorem, a cornerstone result of queuing theory [15] provides the following equation:

$$
\bar{N}_{E V}=\lambda \cdot \bar{P}
$$

where $\lambda$ is the arrival rate of EVs to be charged. This includes a twofold contribution being $\lambda=\lambda_{S M}+\lambda_{W M}$, which takes into account EVs in safe mode (charges are driven by charging 
opportunities), and EVs in warning mode (charges are urgently required), who require a priority queue. This expression is used to plan position of new charging stations and the number of outlets to be deployed at each location.

Field mobility data shown in Fig. 2 suggest that some CS are more relevant than others because of their position. These hot spot CS are able to 'aggregate' charging opportunities of multiple EVs. Simulation results of such aggregations are reported in Fig. 5, where the number of aggregated EVs is represented in logarithmic scale against the arrival rate $\lambda$ of electric vehicles. The $\lambda$ axis is in units of EVs, rather than units of EVs over time, because we consider the same unit of time on the $\mathrm{x}$ and $\mathrm{y}$ axes: if $\mathrm{n}$ vehicles arrive in a specific time period $\mathrm{T}$, then we can aggregate a number of vehicles in the same time T. We consider that a vehicle 'arrives' in the system when a charging opportunity arises or when his battery residual charge is too low. These curves depend on a threshold parameter, the maximum distance that users are willing to walk between the CS and their intended destination. The more users are capable of longer walking distances and the more aggregations are possible, with better utilization of shared resources.

\section{CONCLUSION AND FUTURE WORK}

This work reshapes the problem of charging electric vehicles considering mobility data as key element for optimizing the use of shared charging resources. Unlike previous optimizations, which were dedicated to maximize the utility for DSOs, for the batteries or for the users, we provide an approach that maximizes reuse of intended mobility by exploiting possible charging opportunities. This approach has a positive impact on the environment because it reduces the meta-mobility for vehicle recharge and minimizes user discomfort in the attempt to fit her primary mobility needs. As a future work, we plan to evaluate the impact of our system on sharing charging resources and the aggregating electric vehicles towards specific charging stations to exploit economy of scale as proposed in [16].

\section{ACKNOWLEDGMENT}

The author would like to thank Francesco Randazzo for his quest on the mobility data set and for his preliminary data analysis.

\section{REFERENCES}

[1] C. De Cauwer, J. Van Mierlo, and T. Coosemans, "Energy consumption prediction for electric vehicles based on real-world data," Energies, vol. 8, no. 8, pp. 8573-8593, 2015.
[2] J. Gonder, T. Markel, M. Thornton, and A. Simpson, "Using global positioning system travel data to assess real-world energy use of plugin hybrid electric vehicles," Transportation Research Record: Journal of the Transportation Research Board, no. 2017, pp. 26-32, 2007.

[3] L. Dow, M. Marshall, L. Xu, J. R. Agüero, and H. L. Willis, "A novel approach for evaluating the impact of electric vehicles on the power distribution system," in IEEE PES General Meeting. IEEE, 2010, pp. $1-6$.

[4] G. K. Venayagamoorthy, P. Mitra, K. Corzine, and C. Huston, "Realtime modeling of distributed plug-in vehicles for V2G transactions," in Proc. IEEE Energy Convers. Congr. Expo., San Jose, CA,, Sep. 20-24 2009.

[5] K. Clement-Nyns, E. Haesen, and J. Driesen, "The impact of charging plug-in hybrid electric vehicles on a residential distribution grid," IEEE Transactions on Power Systems, vol. 25, no. 1, pp. 371-380, 2010.

[6] B. D. Lio, A. V. Guglielmi, and L. Badia, "Markov models for electric vehicles: the role of battery parameters and charging point frequency," in 2015 IEEE 20th International Workshop on Computer Aided Modelling and Design of Communication Links and Networks (CAMAD), Sept 2015, pp. 207-210.

[7] M. D. Galus, M. Zima, and G. Andersson, "On integration of plug-in hybrid electric vehicles into existing power system structures," Energy Policy, vol. 38, no. 11, pp. 6736-6745, 2010.

[8] S. D. Jenkins, J. R. Rossmaier, and M. Ferdowsi, "Utilization and effect of plug-in hybrid electric vehicles in the united states power grid," in 2008 IEEE Vehicle Power and Propulsion Conference. IEEE, 2008, pp. 1-5.

[9] N. Rotering and M. Ilic, "Optimal charge control of plug-in hybrid electric vehicles in deregulated electricity markets," IEEE Transactions on Power Systems, vol. 26, no. 3, pp. 1021-1029, 2011.

[10] R. A. Waraich, M. D. Galus, C. Dobler, M. Balmer, G. Andersson, and K. W. Axhausen, "Plug-in hybrid electric vehicles and smart grids: Investigations based on a microsimulation," Transportation Research Part C: Emerging Technologies, vol. 28, pp. 74-86, 2013.

[11] C. Quinn, D. Zimmerle, and T. H. Bradley, "The effect of communication architecture on the availability, reliability, and economics of plug-in hybrid electric vehicle-to-grid ancillary services," Journal of Power Sources, vol. 195, no. 5, pp. 1500-1509, 2010.

[12] C. Guille and G. Gross, "A conceptual framework for the vehicle-to-grid (v2g) implementation," Energy policy, vol. 37, no. 11, pp. 4379-4390, 2009.

[13] M. Wang, H. Liang, R. Zhang, R. Deng, and X. Shen, "Mobility-aware coordinated charging for electric vehicles in vanet-enhanced smart grid," IEEE Journal on Selected Areas in Communications, vol. 32, no. 7, pp. $1344-1360,2014$

[14] R. Trasarti, F. Pinelli, M. Nanni, and F. Giannotti, "Mining mobility user profiles for car pooling," in Proceedings of the 17th ACM SIGKDD international conference on Knowledge discovery and data mining. ACM, 2011, pp. 1190-1198.

[15] M. Harchol-Balter, Performance modeling and design of computer systems: queueing theory in action. Cambridge University Press, 2013.

[16] P. Gallo, F. Randazzo, and I. Gallo, "Fairs for e-commerce: the benefits 2016. of aggregating buyers and sellers," arXiv preprint arXiv:1602.09071, 